\title{
EVALUATION OF PLATELET RICH FIBRIN IN SINUS LIFTING WITH SIMULTANEOUS IMPLANT PLACEMENT \\ Elbaradie $\mathrm{R}^{1} B D S$; Ossman $\mathrm{S}^{2} P h D$; Eldibany $\mathrm{R}^{2} P h D$
}

\begin{abstract}
:
Introduction: Rehabilitation of the edentulous posterior maxilla with dental implants is challenging. The deficient alveolar ridge interferes with implant insertion of adequate length placed in the correct position and with the accurate inclination. The maxillary sinus elevation procedure has become an important preprosthetic surgical procedure for bone creation in the posterior maxilla prior to implant placement. The use of PRF during sinus-lift procedures has been advocated for many years.

Objectives: This study was designed to evaluate the use of platelet rich fibrin (PRF) following sinus lifting with piezosurgery and simultaneous implant placement.

Materials and methods: 7 patients were selected to perform sinus lifting with simultaneous implant placement using PRF as a sole agent and piezosurgery device.

Results: Cone beam computerized tomography 6 months postoperatively showed statistically significant increase in bone height and density. The mean of the newly formed bone height was $(6.55 \pm 1.14 \mathrm{~mm})$. The mean postoperative bone height measured from the floor of the maxillary sinus and alveolar crest was $(11.35 \pm 0.56 \mathrm{~mm})$. The mean of the newly formed bone density was $507 \mathrm{HU}$. The mean of the postoperative bone density around the implants after 6 months was $(547.71 \pm 188.42 \mathrm{HU})$. The mean marginal bone loss was $(0.82 \pm 0.25 \mathrm{~mm})$.
\end{abstract}

Conclusion: PRF could be successfully used as a sole agent for bone regeneration in lateral sinus lifting with simultaneous implant placement.

Key words: lateral sinus lifting, platelet rich fibrin, Piezosurgery, implants, bone density.

1- Dentist, Alexandria Dental Research Center, Ministry of Health, Egypt.

2- Professor of Oral and Maxillofacial Surgery, Faculty of Dentistry, Alexandria University, Egypt.

\section{INTRODUCTION}

Replacement of lost natural teeth by osseointegrated implants has been represented as one of the most significant advances in prosthetic dentistry. Endosseous dental implants are widely inserted to restore oral function, including mastication and speech, as well as for aesthetic improvement in partly or completely edentulous patients $(1,2)$.

However, insufficient height and/or width of the alveolar ridge when placing implants for oral rehabilitation in the atrophied maxilla is a challenge. Ridge resorption and sinus pneumatization in the posterior maxilla, compounded with poor quality of bone, can compromise implant rehabilitation of the patient (3).

The maxillary sinus elevation procedure has become an important preprosthetic surgical procedure for the creation of bone volume in the edentulous posterior maxilla for the placement of dental implants (4).

The most widely used techniques for maxillary sinus floor elevation is the classical lateral antrostomy introduced by Tatum in 1976. Lateral window may be performed using a 1- or 2-step approach. Implants are installed simultaneously with the bone graft (1-stage lateral antrostomy) or after a delay to allow for bone healing (2-stage lateral antrostomy) (5).

The most common intraoperative complication with these surgical approaches is the perforation of the Schneiderian Membrane. Wallace et al (6) stated that the membrane perforation rate has been reduced from the average reported rate of $30 \%$ with rotary instrumentation to $7 \%$ using the piezoelectric technique. The piezosurgery device provides a clear surgical site, as it maintains a blood-free surgical field during bone cutting. This allows improved visualization of the surgical area. A very small amount of pressure is applied which allows a very precise cut. The typical cavitation effect induces a hydropneumatic pressure in the physiological saline solution that contributes to atraumatic sinus membrane elevation (5).

Although sinus elevation using autogenous bone graft is considered to be the gold standard, many researchers have attempted to modify this procedure, because of the morbidity associated with bone harvesting. Various nonautogenous substitutes, such as xenogenic, allogenic and some artificial materials have been developed to reduce the risks associated with autogenous bone grafts. However, the use of xenogenic or allogenic materials also induces the risk of disease transmission and have been found to be insufficient for bone regeneration, and artificial bone grafts have been found to be insufficient for osteogenic regeneration (7-9).

The use of blood preparations such as plasma concentrates or fibrin glue might be an interesting option to improve this approach. Platelet rich fibrin has many characteristics that make it suitable for application as a filling material for sinus floor augmentation. It is an autologous fibrin matrix that is rich in platelets, leukocytes and growth factors. It has moderate strength, is easy to handle and promotes healing of the sinus membrane and bone (7).

Therefore, the present study was designed to evaluate the effect of PRF on bone regeneration in sinus lifting using piezosurgery and simultaneous implant placement. 


\section{MATERIALS AND METHODS}

\section{Patients Selection and Evaluation:}

This study was conducted on seven patients selected from the Out-patient Clinic of the Oral and Maxillofacial Surgery Department, Faculty of Dentistry, Alexandria University. Patients were seeking prosthetic rehabilitation of missing maxillary posterior teeth and suffering from locally resorbed maxillary ridge, presenting a problem for dental implant insertion. All patients were informed about the aim of the designed study and a written consent was obtained.

The inclusion criteria of this study were; patients having missing maxillary posterior teeth, the vertical height between the alveolar crest and the floor of the maxillary sinus ranged between $4 \mathrm{~mm}$ and $7 \mathrm{~mm}$, adequate inter-arch relation and inter-occlusal space that could accommodate the implant abutment and the future restoration, free from acute or chronic maxillary sinusitis as confirmed by Ear Nose And Throat (ENT) examination, recipient site free from any pathology, adequate oral hygiene, patients psychologically accepting dental implants and the involved procedure. While the exclusion criteria were; systemic diseases that contra-indicate implant placement such as uncontrolled diabetes mellitus, bleeding disorders, serious osseous disorders, cardiac arrhythmia, patients on pacemakers and mental disorders, heavy smoking and alcoholism, parafunctional habits such as bruxism and clenching.

Preoperative clinical examination was performed for all patients: Patients data were collected; name, gender and age, medical and dental history were taken and the oral mucosa of the edentulous area was examined for color, texture, firmness and thickness. All patients were clinically examined by ear nose and throat (ENT) specialist. Also, preoperative evaluation for all patients included panoramic $\mathrm{x}$-ray and cone beam computerized tomography (CBCT), to evaluate the residual ridge height and width, the anatomy of the maxillary sinus, and to evaluate the condition of bone, as shown in (figure 1).

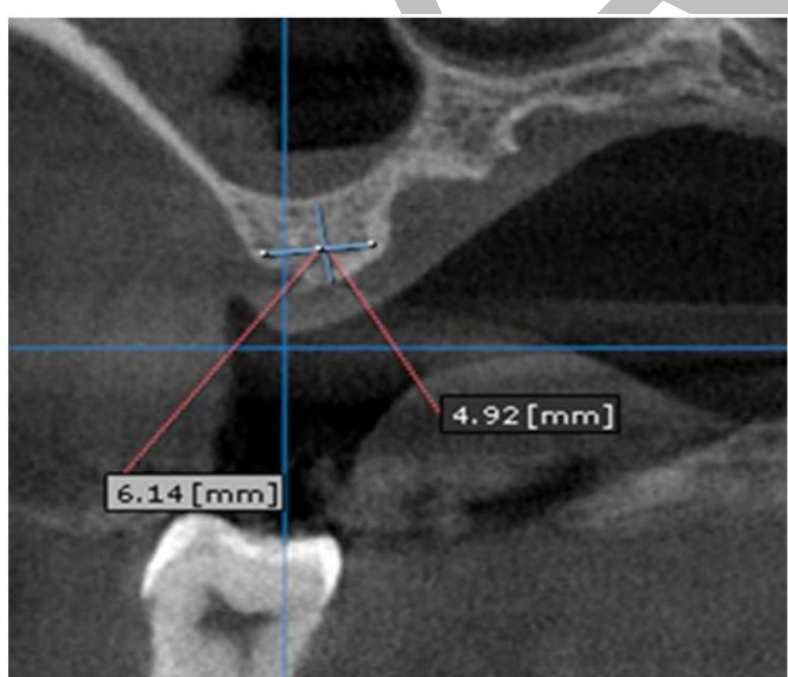

Fig.1: Preoperative CBCT (sagittal cut) showing maxillary posterior alveolar ridge deficiency.

\section{Informed Consent:}

Appropriate institutional ethical clearance and written informed consent were obtained.

\section{Surgical procedure:}

Preoperative oral sedation diazepam $10 \mathrm{mg}$ (Valium $10 \mathrm{mg}$ tab, Roche Pharmaceutical Co., Basel, Switzerland) tablets given 1 hour prior to surgery. Also, preoperative oral antibiotic prophylaxis one hour before surgery was given Amoxicillin (Amoxil 1gm capsule, Smithline Beecham Pharmaceutical Co., Bentford, England ) $2 \mathrm{mg}$ capsule and $0.12 \%$ chlorohexidine HCL (Hexitol Mouth wash, The arab drug Co., Cairo, Egypt) mouth wash was used to rinse for 30 seconds before the surgical operation. Ephedrine spray/nasal drops $1.0 \%$ (Otrivin spray/nasal Drops $10 \mathrm{ml}$, Novartis Pharma AG, Basle, Switzerland) was used as a vasoconstrictor in order to decongest the nasal passages to minimize intraoperative bleeding.

The oral mucosa was painted and swabbed thoroughly with antiseptic povidone iodine solution $10 \%$ (Betadine, Nile Company for Pharmaceuticals, Cairo, Egypt) to render the surgical field free from microorganisms. Under local anesthesia, a pyramidal full thickness mucoperiosteal flap was designed distal to the canine area with a crestal incision placed palatally in the edentulous area and vertical extension to the buccal vestibule using Bard Parker blade number 15.The flap was reflected to expose the alveolar bone.

A lateral window was performed using the piezosurgery device (Silfadent, Italy): Initially a bony window was cut using insert SB PO200 on a power 30/ 100 vibration microns, then the window was freed using SB PO400 on a power 18/100 vibration microns, as shown in (figure 2).

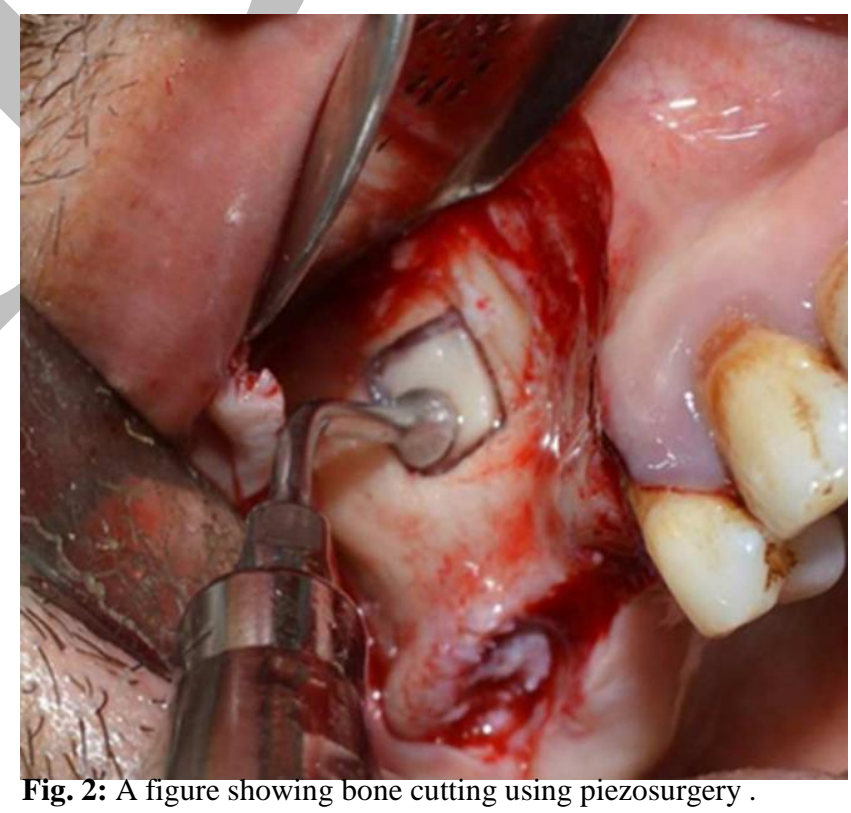

Finally the sinus membrane was dissected and elevated along with the trap door using SB PO321 on a power 20/100 vibration microns.

Drilling at the implant site was performed using an implant micromotor and Neobiotech implant drilling kit, implants (IS1 bone level Neobiotech Implant System, 
Korea.) were used in this study. A platform switching is present between the implant and the abutment, which minimizes the microgap and maximizes the biologic width in order to minimize bone loss. It has a tapered body with powerful threads at the apex to facilitate implant insertion. They were inserted using a ratchet wrench until the implant body was flushed with bone surface.

\section{PRF preparation:}

Platelet rich fibrin (PRF) was prepared according to the protocol developed by Choukroun et al (10) (3000 RPM for $10 \mathrm{~min}$ ) using a table centrifuge.

During surgery $20 \mathrm{ml}$ of whole blood was obtained from the brachial vein, the blood was transferred and divided into two $10 \mathrm{ml}$ sterile glass tubes without anti-coagulant. Immediate centrifugation was performed using a table centrifuge. The coagulation cascade starts during centrifugation and the blood is divided into 3 parts in the tube: serum at the upper part, red blood cells (RBCs) at the bottom and PRF in between which is separated and used as the augmentation material.

PRF was prepared and placed over the implants tented under the maxillary sinus membrane, as shown in (figure 3 ). Then the flap was repositioned and sutured using 3-0 black silk suture material.

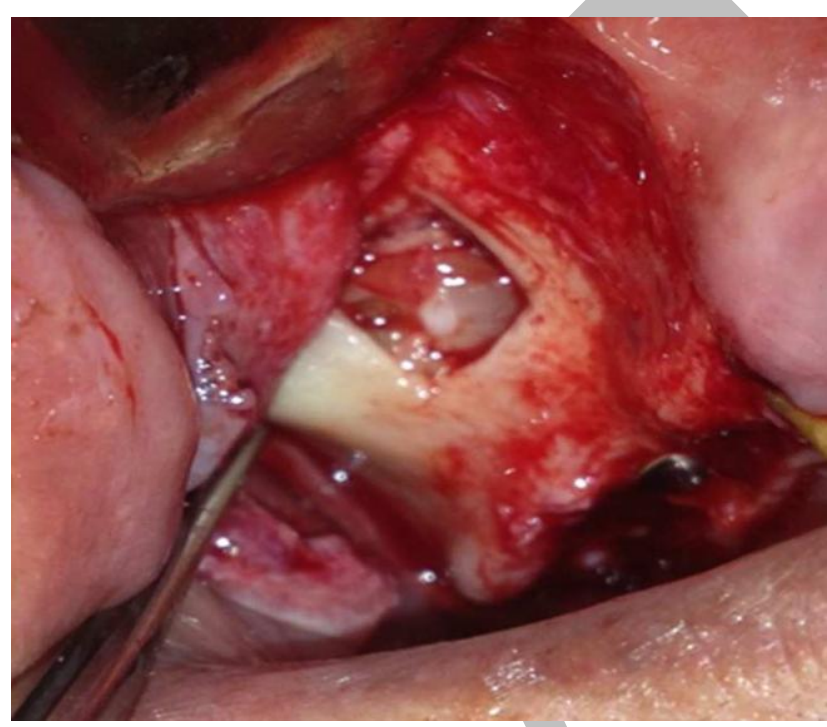

Fig. 3: A picture showing PRF in the maxillary sinus with simultaneous implant placement.

All patients were advised to; apply cold packs extra orally intermittently and avoid hot food on the first day, apply hot packs on the second day, avoid eating hard food at the surgical site, chlorhexidine mouth wash was started on the 2nd post-operative day 3 times daily for 10 weeks. Broad spectrum antibiotic Amoxicillin $875 \mathrm{mg}+$ Clavulanic acid $125 \mathrm{mg}$ tablets (Augmentin $1 \mathrm{gm}$ Smithline Beecham Pharmaceutical Co., Bentford, England) in combination with metronidazole $500 \mathrm{mg}$ capsule (Amrizole $500 \mathrm{mg}$ tablets, Amriya Pharmaceutical Industries, Egypt) twice daily for 5 days to avoid post-operative infection. Non-steroidal antiinflammatory analgesic in the form of diclofenac potassium
$50 \mathrm{mg}$ tablets (Cataflam $50 \mathrm{mg}$ tablets, Novartis Pharma AG,Basle, Switzerland) 3 times daily for 7-10 days to avoid the possibility of inflammation, oedema and pain. Ephidrine nasal drops (Otrivin spray/nasal Drops $10 \mathrm{ml}$, Novartis Pharma AG, Basle, Switzerland) 3-5 times daily for 5 days. Sutures were removed after 10 days.

\section{Postoperative evaluation}

All patients were examined the day after surgery then weekly for the first month postoperatively, then on intervals of 1,4 and 6 months postoperatively. The clinical parameters of importance for determination of implant success included: Absence of pain, tenderness, discomfort, wound dehiscence, implant mobility or any other complications related to the sinus lifting or implant placement. Pain and discomfort were examined using visual analogue scale (VAS). Patients were asked to assess the level of their average pain by placing a mark on a horizontal line that was $10 \mathrm{~cm}$ long (11). Tenderness by palpation and swelling was measured by inspection.

Immediate CBCT were obtained to evaluate the surgical procedure and implant placement, as shown in (figure 4).

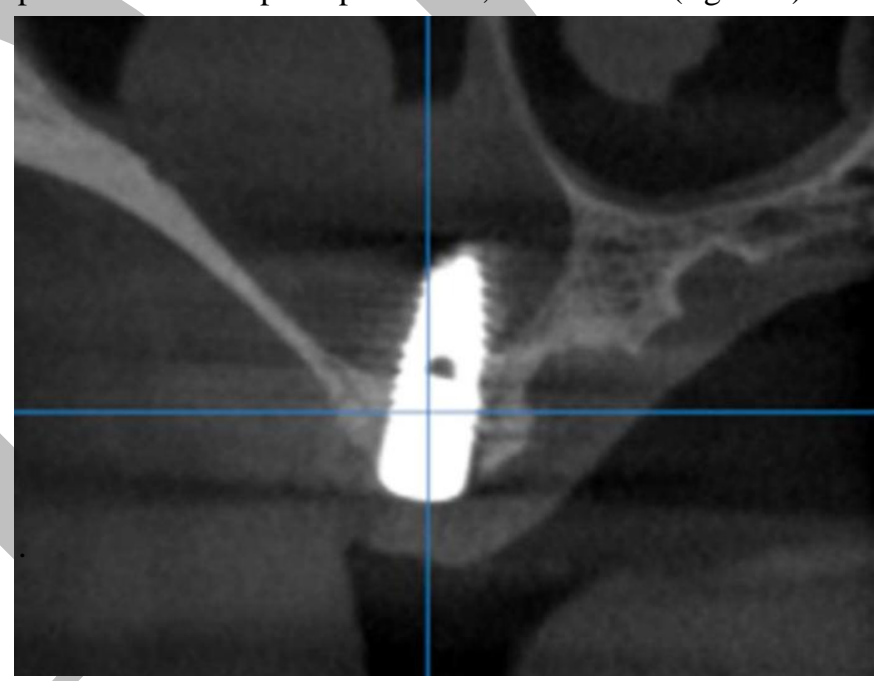

Fig. 4: Immediate post-operative CBCT (sagittal cut) to evaluate the surgical procedure and implant placement.

Periapical $\mathrm{x}$-rays were obtained immediately postoperative and on intervals of 1 and 4 month. CBCT was obtained 6 month postoperatively for all patients to measure bone density, marginal ridge resorption and the height of the newly formed bone, as shown in (figure 5).

CBCT measurements were performed using On Diamond 3DApp-DBM software system (Cypernet, Korea) where the bone height could be measured and through which the bone density is calculated directly in HU.

\section{RESULTS}

In this study, seven sinus floor augmentations were performed on seven patients. The selected patients were 2 males and 5 females, and their age ranged from 28-60 years with a mean age $(39.86 \pm 13.02$ years). The mean height of the alveolar ridge from the marginal crest to floor of the maxillary sinus was $5.84 \mathrm{~mm} \pm 0.79 \mathrm{~mm}$ (Range: $4.49-6.8$ $\mathrm{mm}$ ), as shown in (table 1). 


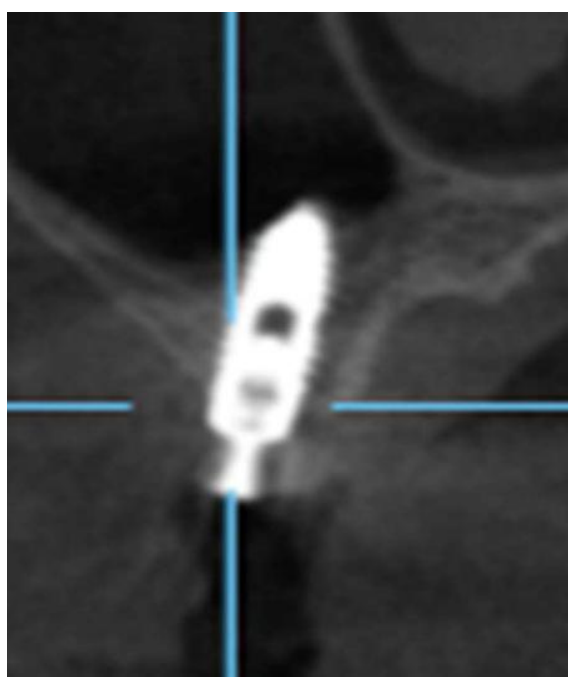

Fig. 5: CBCT 6 months post-operatively (sagittal cut) showing new bone formation.

Table (1): Description regarding age, gender, implant location, preoperative bone width and height and implant length and diameter.

\begin{tabular}{|c|c|c|c|c|c|c|c||}
\hline $\begin{array}{c}\text { Case } \\
\#\end{array}$ & Gender & Age & $\begin{array}{c}\text { Implant } \\
\text { location }\end{array}$ & $\begin{array}{c}\text { Preoperative } \\
\text { bone } \\
\text { height(mm) }\end{array}$ & $\begin{array}{c}\text { Preoperative } \\
\text { bone } \\
\text { width(mm) }\end{array}$ & $\begin{array}{c}\text { Implant } \\
\text { length (mm) }\end{array}$ & $\begin{array}{c}\text { Implant } \\
\text { diameter } \\
(\mathbf{m m})\end{array}$ \\
\hline 1 & $\mathrm{~F}$ & 28 & Premolar & 6.8 & 5.5 & 10 & 3.5 \\
\hline 2 & $\mathrm{~F}$ & 28 & Molar & 6.5 & 4.7 & 10 & 3.5 \\
\hline 3 & $\mathrm{~F}$ & 30 & Molar & 5.2 & 6.35 & 13 & 3.5 \\
\hline 4 & $\mathrm{M}$ & $\mathbf{3 8}$ & Premolar & 4.49 & 6.2 & 10 & 3.5 \\
\hline 5 & $\mathrm{~F}$ & 60 & Molar & 5.1 & 4.5 & 10 & 3.5 \\
\hline 6 & $\mathrm{~F}$ & 55 & Molar & 6.6 & 4.7 & 10 & 3.5 \\
\hline 7 & M & 40 & Molar & 5.8 & 4.4 & 13 & 3.5 \\
\hline
\end{tabular}

Seven implants were placed in the maxillary posterior teeth, 5 at the molar region and 2 in premolar region. The patients received implants with length ranged from $10.0-$ $13.0 \mathrm{~mm}$. All the placed implants were $3.5 \mathrm{~mm}$ in diameter.

\section{1-Pain index:}

Pain index recorded its highest ranging from 1-3 with a mean of $(2.0 \pm 0.82)$ during the 1 st post-operative day, $0-2$ with a mean of $(1.00 \pm 0.82)$ during the 1st post-operative week, $0-1$ with a mean of $(0.29 \pm 0.49)$ during the 2 nd postoperative week. While no pain was recorded since the 3rd week postoperatively till the rest of the follow up period. It was statistically significant starting from the 1 st week postoperatively compared to the 1 st post-operative day ( $\mathrm{p} \leq$ $0.05)$.

\section{2- Tenderness}

Tenderness was present only in 2 patients during the 1 st post-operative day and absent in all patients during the rest of the follow up period. Swelling was observed in 3 patients during the 1st post operative day, and resolved completely in all cases during the 1st post-operative week with the absence of wound dehiscence in all cases.

\section{3-Nasal obstruction and nasal bleeding}

Nasal obstruction was a complaint for 3 patients during the 1st postoperative day. Only 2 patients had complained during the 1st and 2nd postoperative weeks. Nasal bleeding was absent in all cases. All implants were clinically stable and remained stable during abutment insertion.

\section{4- Radiographic evaluation}

CBCT 6 months postoperatively showed statistically significant increase in bone height and density. The newly formed bone height ranged from $5.12-8.10 \mathrm{~mm}$ with a mean of $(6.55 \pm 1.14 \mathrm{~mm})$. The mean postoperative bone height measured from the floor of the maxillary sinus and alveolar crest was $(11.35 \pm 0.56 \mathrm{~mm})$ with a range from $10.40-11.90 \mathrm{~mm}$. A significant change was indicated compared to the preoperative bone height, with a mean percentage of change of $97.05 \%$, as shown in (table 1, figure 6). The mean newly formed bone density was $507 \mathrm{HU}$ and ranged from 250- $798 \mathrm{HU}$. The mean postoperative bone density around the implants after 6 month was $(547.71 \pm$ $188.42 \mathrm{HU})$ and ranged from $(245.0-803.0 \mathrm{HU})$, which changed significantly compared to the preoperative bone density, with a mean percentage of change $88.79 \%$, as shown in (table 2, figure 6).

The mean marginal bone loss was $0.82 \pm 0.25 \mathrm{~mm}$ with a range of $0.48-1.20$ at the 6 th month postoperatively.
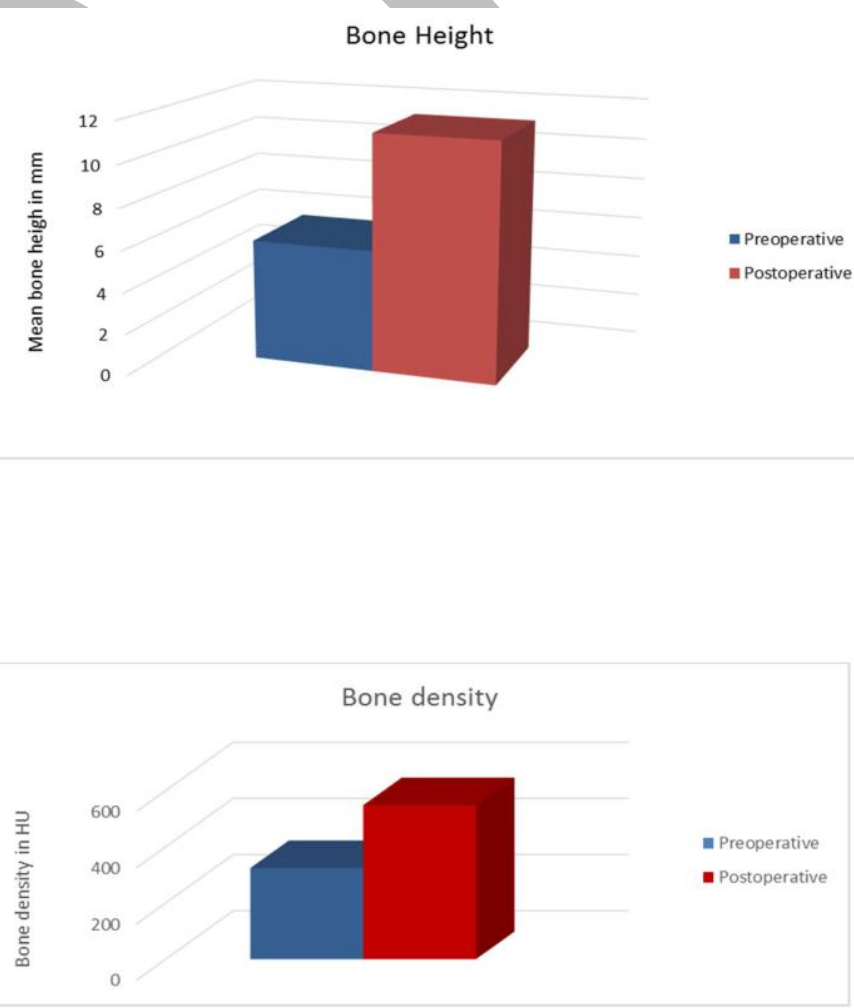

Fig. 6: Charts comparing between preoperative and postoperative bone height and bone density.

Table (2): Comparison between preoperative and postoperative bone height.

\begin{tabular}{|c|c|c|c|}
\hline & \multicolumn{3}{|c|}{ Bone height } \\
\hline & $\begin{array}{c}\text { Preoperative } \\
(\mathbf{n}=7)\end{array}$ & $\begin{array}{c}\text { Postoperative } \\
(n=7)\end{array}$ & $\%$ of change \\
\hline Min. - Max. & $4.49-6.80$ & $10.40-11.90$ & $60.88-128.85$ \\
\hline Mean \pm SD & $5.84 \pm 0.79$ & $11.35 \pm 0.56$ & $97.05 \pm 25.70$ \\
\hline Median & 5.80 & 11.30 & 103.45 \\
\hline$t(p)$ & \multicolumn{2}{|c|}{$16.729^{*}\left(<0.001^{*}\right)$} & \\
\hline
\end{tabular}

t: Paired t-test *: Statistically significant at $\mathrm{p} \leq 0.05$ 
Table 3: Comparing between preoperative and 6 months bone density around implants

\begin{tabular}{|c|c|c|c|}
\hline & \multicolumn{3}{|c|}{ Bone density } \\
\hline & $\begin{array}{l}\text { Preoperative } \\
\quad(\mathbf{n}=7)\end{array}$ & $\begin{array}{l}\text { Postoperative } \\
\quad(\mathbf{n}=7)\end{array}$ & $\%$ of change \\
\hline Min. - Max. & $142.0-591.0$ & $245.0-803.0$ & $12.39-171.83$ \\
\hline Mean $\pm \mathrm{SD}$ & $324.29 \pm 171.43$ & $547.71 \pm 188.42$ & $88.79 \pm 59.05$ \\
\hline Median & 280.0 & 540.0 & 65.87 \\
\hline$t(p)$ & \multicolumn{2}{|c|}{$5.301^{*}\left(0.002^{*}\right)$} & \\
\hline
\end{tabular}

\section{DISCUSSION}

Bone resorption and pneumatization of the maxillary sinus, following tooth extraction, are common in the posterior maxilla. They may cause both a quantitative reduction and a qualitative deterioration of bone that leads to inadequate bone dimension for proper size/length implant placement (12).

The present study was designed to evaluate the use of PRF as a sole agent in the augmentation of resorbed edentulous posterior maxilla following sinus lift using piezosurgery and simultaneous implant placement.

The selected patients were systemically free from any disease or systemic condition to avoid any systemic influence on bone formation or bone resorption This was in accordance with an 11 years retrospective study performed by Moy et al (13) in 2005 that included systemic disease as high risk factor for implant failure.

Also, heavy smokers were excluded from this study. This was in agreement with a study by Holahan et al (14) in 2008, where they concluded that implants in patients who were smokers during the time of implant placement were 2.6 times more likely to fail compared to implants placed in patients who did not smoke.

In this study, patients with parafunctional habits such as bruxism and clenching were also excluded and a strict oral hygiene was followed by all patients during the preoperative and postoperative follow up. This was in agreement with a study by Porter and von Fraunhofer (15) in 2005.

Also, patients in this study were selected free from any sinus pathosis after ear nose and throat (ENT) consultation. This is in agreement with a study conducted by Torretta et al (16) in 2013, which recommended that a careful multitasking preoperative management, including an ENT assessment is useful in patients undergoing sinus membrane elevation.

In the current study antibiotics were administrated preoperatively and postoperatively, as maxillary sinus augmentation and implant placement procedures run a risk of introducing new bacteria into the sinus. The antibiotic administration has been demonstrated not only to minimize the incidence of postoperative infection but also to significantly reduce the rate of implant failure as stated by Trieger (17) in 1999, Laskin et al (18) in 2000 and supported by Sharaf et al (19) in 2011.

The initial bone height from the alveolar crest till the floor of the maxillary sinus that received the implants ranged from $4.90-6.80 \mathrm{~mm}($ Mean $\pm \mathrm{SD}, 5.84 \pm 0.79)$. This is supported by a recent study conducted by Mardinger et al (21) in 2011 to compare the radiographic dimensional changes of sinus graft height above and between placed implants, and evaluate the factors affecting these changes with 2 different grafting materials and both combination. They reported $92 \%$ success rate in patients with 1 to $3 \mathrm{~mm}$ of residual vertical bone height compared with $98.7 \%$ with residual bone height of more than $4 \mathrm{~mm}$.

The lateral window approach was used in this study for sinus membrane elevation with a $100 \%$ success rate with no implant failure until the 6th month postoperatively. This is in agreement with Wallace and Froum (22) in 2003 that stated in their study a survival rate of $91.8 \%$.

Piezoelectric surgery was used to access the maxillary sinus through the lateral window osteotomy and sinus membrane elevation to protect the soft tissues and minimize patient discomfort. This is in agreement with Al-Dajani (23) in 2014 and others (24) who concluded that the use of piezoelectric surgery allows adequate sinus lift while protecting soft tissues and minimizing patient discomfort.

Thus the use of piezoelectric devices seems to simplify sinus lift surgical procedures and to allow greater predictability with less postoperative complications.

This is in agreement with Carini et al (25) in 2014 where they reported that bone tissue healing showed a reduced rate of bone loss with piezoelectric instruments than with conventional devices, as well as a better healing quality by reducing patient's postoperative morbidity.

PRF was used in this study because it is a fibrin-matrix in which platelet, cytokines and cells are entrapped and released after a certain time and can serve as resorbable membrane, it is considered to be a healthy biomaterial, and was initially used in oral implantology by its promoters, and presently, its application has been advocated in various disciplines of dentistry (26).

PRF concentrates most platelets and more than half of live and functional leukocytes from the blood harvest (27) which releases high amounts of growth factors (such as transforming growth factor- $\beta 1$ [TGF $\beta-1]$, plateletderived growth factor-AB [PDGF-AB]. Thus, it is an autologous and inexpensive material, which is considered as an optimized blood clot, as stated by Tajima et al (7) in 2013.

In the present study no wound dehiscence was present post operatively in any of the cases and radiographic evaluation by CBCT 6 month postoperatively revealed the absence of any fluid level or inflammatory process.

Nasal bleeding was absent in all cases postoperatively during the follow up period. While pain and discomfort scale significantly changed after the first day. Swelling was present in 3 cases on the first day only and nasal obstruction was a postoperative complain in 3 cases on the first day, with its absence in all cases for the rest of the follow up period.

This coincides with Carini et al (24) in 2014, in their study where they observed better healing quality by reducing patient's postoperative morbidity when using piezosurgery.

Also, in agreement with, Dohan et al (28) in 2006 who stated that PRF might decrease many harmful effects due to 
inflammatory processes that are inherent to the surgical act itself, mainly by correcting certain destructive and noxious excesses during the healing process of wounded tissues.

In addition, He et al (29) in 2009 in an in vitro study reported the superiority of PRF in the expression of alkaline phosphatase and induction of mineralization. They remarked that PRF released autologous growth factors gradually and expressed stronger and more durable effect on proliferation and differentiation of osteoblasts than PRP.

In the present study, CBCT obtained 6 months after surgery revealed sufficient newly formed bone in all treated cases, elevating the sinus membrane showing repneumatization around the implants apices. The new bone showed adaptation in shape and volume with the repneumatized maxillary sinus with a decreased noted visibility of the original sinus floor. The apex of the implants remained surrounded with bone radiographically. This indicated the stability of the newly formed bone and bone maturation. The sinus membrane is maintained elevated and the bone gained is preserved.

This is in agreement with a study conducted by Simonpieri et al (30) in 2011, where they observed that the final level of the new sinus floor was always in continuation with the implant apical end.

In this study, there was a significant change in bone height. The new bone height formed measured from the floor of the maxillary sinus was $5.12-8.10 \mathrm{~mm}($ Mean $=$ $6.55 \pm 1.14)$. The total over all bone height at the end of the procedure at a 6 month follow up period was $10.40-11.90$ $\mathrm{mm}($ Mean $=11.35 \pm 0.56)$, with a mean percentage of change of $97.05 \%$, which relatively corresponds to the actual length of the inserted implants. Because the implants served as a tent to maintain the height of the bone healing space the final vertical bone height was dependent on the implant length.

This is in agreement with a study conducted by Mazor et al (27) in 2009, where the radiographic analysis showed that the final bone gain was always significant with the implant length.

Norton \& Gamble (31) in 2001 suggested that Bone density can be evaluated using Hounsfield units, which are directly related to tissue attenuation coefficients taken from CT. And recorded the mean bone density was $682 \mathrm{HU}$ for 139 sites. While, the mean bone densities in the anterior maxilla and the posterior maxilla were 696, and $417 \mathrm{HU}$ respectively.

In the present study, the density of the new bone formed around implants after 6 month ranged from 250.0 - $798 \mathrm{HU}$ $($ Mean $=507.0 \pm 191.24)$, which is comparable to that of bone normally present in the maxilla, with a significant change in bone density around the implants comparing the preoperative bone density around the implants that ranged from 142.0 - 591.0 HU (Mean = 324.29 \pm 171.43) and bone density around the implants 6 month postoperatively that ranged from $245.0-803 \mathrm{HU}($ Mean $=547.71 \pm 188.42)$, with a mean percentage of change $88.79 \%$.

This is in agreement with the results of Sogo et al (32) in 2012, where they studied the bone density of the posterior maxilla in 30 patients and concluded that the bone in the posterior maxilla was classified as D3 (350-850 HU) or D4 (150-350 HU) according to Misch's classification, comprising $50 \%$ and $32 \%$ of the entire regions, respectively.

The radiographic evaluation 6 month postoperatively using CBCT measures showed that the marginal ridge resorption ranged from $0.48-1.20 \mathrm{~mm}$ (Mean $=0.82 \pm$ $0.25)$. This coincides with a study that mentioned the mean marginal bone loss within the first year was $1.2+/-0.7 \mathrm{~mm}$ conducted by Nedir et al (33).

\section{CONCLUSIONS}

The use of piezosurgery is safe and reduces both intraoperative and postoperative complications. Platelet rich fibrin is an autologous and inexpensive material. Sinus floor augmentation with PRF as a sole material is a secure and reliable method for promoting natural bone formation prior to implant placement. It does not require placement of additional bone grafting material.

\section{STATEMENT OF CONFLICT OF INTEREST}

The authors declare that they have no conflicts of interest.

\section{REFERENCES}

1. Liu Q, Liu X, Liu B, Hu K, Zhou X, Ding Y. The effect of low-intensity pulsed ultrasound on the osseointegration of titanium dental implants. Br J Oral Maxillofac Surg. 2012;50(3):244-50.

2. Viswambaran M, Arora V, Tripathi RC, Dhiman RK. Clinical evaluation of immediate implants using different types of bone augmentation materials. Med J Armed Forces India. 2014;70(2):154-62.

3. Calandriello R, Tomatis M. Simplified treatment of the atrophic posterior maxilla via immediate/early function and tilted implants: a prospective 1-year clinical study. Clin Implant Dent Relat Res 2005;7(1):S1-12.

4. M. Rapani, C. Rapani. Sinus floor lift and simultaneous implant placement: A retrospective evaluation of implant success rate. Indian Journal of Dentistry 2012;3(3):132-8.

5. Baldi D, Menini M, Pera F, Ravera G, Pera P. Sinus floor elevation using osteotomes or piezoelectric surgery. Int J Oral Maxillofac Surg. 2011;40(5):497503.

6. Wallace SS, Tarnow DP, Froum SJ, Cho SC, Zadeh $\mathrm{HH}$, Stoupel J et al. Maxillary sinus elevation by lateral window approach: evolution of technology and technique. J Evid Base Dent Pract 2012;1:161-71.

7. Tajima N, Ohba S, Sawase T, Asahina I. Evaluation of sinus floor augmentation with simultaneous implant placement using platelet-rich fibrin as sole grafting material. Int J Oral Maxillofac Implants. 2013;28(1):77-83.

8. Khairy NM, Shendy EE, Askar NA, El-Rouby DH. Effect of platelet rich plasma on bone regeneration in maxillary sinus augmentation (randomized clinical trial). Int. J. Oral Maxillofac. Surg. 2013; 42: 249-55.

9. Hallman M, Lundgren S, Sennerby L. Histologic analysis of clinical biopsies taken 6 months and 3 years after maxillary sinus floor augmentation with $80 \%$ bovine hydroxyapatite and $20 \%$ autogenous bone mixed 
with fibrin glue. Clin Implant Dent Relat Res. 2001;3(2):87-96.

10. Choukroun J, Diss A, Simonpieri A, Girard MO, Schoeffler C, Dohan SL et al. Platelet-rich fibrin (PRF): a second-generation platelet concentrate. Part V: histologic evaluation of PRF effect on bone allograft maturation in sinus lift. Oral Surg Oral Med Oral Pathol Oral Radiol Endod. 2006;101(3):299-303.

11. Ghanbari JA, Makvandi A, Pazouki A. Quality of sleep for hospitalized patients in Rasoul-Akram hospital. Med J Islam Repub Iran. $2014 ; 21(28): 73$.

12. Soardi CM, Spinato S, Zaffe D, Wang HL. Atrophic maxillary floor augmentation by mineralized human bone allograft in sinuses of different size: A histologic and histomorphometric analysis. Clin Oral Implants Res. 2011;22(5):560-6.

13. Moy PK, Medina D, Shetty V, Aghaloo TL. Dental implant failure rates and associated risk factors. Int $\mathrm{J}$ Oral Maxillofac Implants. 2005;20:569-77.

14. Holahan CM, Koka S, Kennel KA, Weaver AL, Assad DA, Regennitter FJ, et al. Effect of osteoporotic status on the survival of titanium dental implants. Int J Oral Maxillofac Implants. 2008;23(5):905-10.

15. Porter JA, Von Fraunhofer JA. Success or failure of dental implants? A literature review with treatment considerations. Gen Dent. 2005;53(6):423-32.

16. Torretta S, Mantovani M, Testori T, Cappadona M, Pignataro L. Importance of ENT assessment in stratifying candidates for sinus floor elevation: a prospective clinical study. Clin Oral Implants Res. 2013;24 (100):57-62.

17. Trieger N. Antibiotics and anti-inflammatory agents in dental implantology. Implant Dent. 1999; 8:343-6.

18. Laskin DM, Dent CD, Morris HF, Ochi S, Olson JW. The influence of preoperative antibiotics on success of endosseous implants at 36 months. Ann Periodontol. 2000;5(1):166-74.

19. Sharaf B, Dodson TB. Does the use of prophylactic antibiotics decrease implant failure? Oral Maxillofac Surg Clin North Am. 2011;23(4):547-50.

20. Bornstein MM, Scarfe WC, Vaughn VM, Jacobs R. Cone beam computed tomography in implant dentistry: a systematic review focusing on guidelines, indications, and radiation dose risks. Int J Oral Maxillofac Implants. 2014; 29:55-77.

21. Mardinger O, Chaushu G, Sigalov S, Herzberg R, Shlomi B, Schwartz-Arad D. Factors affecting changes in sinus graft height between and above the placed implants. Oral Surg Oral Med Oral Pathol Oral Radiol Endod. 2011; 11:6-11.

22. Wallace SS, Froum SJ. Effect of maxillary sinus augmentation on the survival of endosseous dental implants. A systematic review. AnnPeriodontol 2003;8:328-343.

23. Al-Dajani M. Recent Trends in Sinus Lift Surgery and Their Clinical Implications. Clin Implant Dent Relat Res. 2014 Oct 2.

24. Pereira CC, Gealh WC, Meorin-Nogueira L, GarciaJúnior IR, Okamoto R. Piezosurgery applied to implant dentistry: clinical and biological aspects. J Oral Implantol. 2014;40:401-8.

25. Carini F, Saggese V, Porcaro G, Baldoni M. Piezolelectric surgery in dentistry: a review. Minerva Stomatol. 2014;63(1-2):7-34.

26. Dohan DM, Choukroun J, Diss A, Dohan SL, Dohan AJ, Mouhyi J et al. Platelet-rich fibrin (PRF): a secondgeneretion platelet concentrate. Part II: platelet-related biologic features. Oral Surg Oral Med Oral Pathol Oral Radiol Endod. 2006;101(3):e45-50.

27. Mazor Z, Horowitz RA, Del Corso M, Prasad HS, Rohrer MD, Dohan Ehrenfest DM. Sinus floor augmentation with simultaneous implant placement using Choukroun's platelet-rich fibrin as the sole grafting material: a radiologic and histologic study at 6 months. J Periodontol. 2009;80(12):2056-64.

28. Dohan DM, Choukroun J, Diss A, Dohan SL, Dohan AJ, Mouhyi J et al. Platelet-rich fibrin (PRF): a secondgeneretion platelet concentrate. Part III: leucocyte activation: a new feature for platelet concentrate?. Oral Surg Oral Med Oral Pathol Oral Radiol Endod. 2006;101(3):e51-5.

29. He L, Lin Y, Hu X, Zhang Y, Wu H. A comparative study of platelet-rich fibrin (PRF) and platelet-rich plasma (PRP) on the effect of proliferation and differentiation of rat osteoblasts in vitro. Oral Surg Oral Med Oral Pathol Oral Radiol Endod. 2009;108(5):707-13.

30. Simonpieri A, Choukroun J, Del Corso M, Sammartino G, Dohan Ehrenfest DM. Simultaneous sinus-lift and implantation using microthreaded implants and leukocyte- and platelet-rich fibrin as sole grafting material: a six-year experience. Implant Dent. 2011;20(1):2-12.

31. Norton MR, Gamble C. Bone classification: An objective scale of bone density using the computerized tomography scan. Clin Oral Implants Res 2001;12:79-84.

32. Sogo M, Ikebe K, Yang TC, Wada M, Maeda Y. Assessment of bone density in the posterior maxilla based on Hounsfield units to enhance the initial stability of implants. Clin Implant Dent Relat Res. 2012; 14:e183-7.

33. Nedir R, Bischof M, Vazquez L, Szmukler-Moncler S, Bernard JP. Osteotome sinus floor elevation without grafting material: A 1-year prospective pilot study with ITI implants. Clin Oral Implants Res 2006;17:679-86. 